\title{
Discours
}

Revue de linguistique, psycholinguistique et

informatique. A journal of linguistics, psycholinguistics

and computational linguistics

$8 \mid 2011$

Approches fonctionnelles de la structuration des textes

\section{Contribution des trois emplois de à la fin à la structuration du discours : le temporel, l'aspectuel, le « reformulatif »}

\section{Christiane Marque-Pucheu}

\section{(2) OpenEdition}

\section{Journals}

Édition électronique

URL : http://journals.openedition.org/discours/8468

DOI : $10.4000 /$ discours.8468

ISSN : 1963-1723

Éditeur :

Laboratoire LATTICE, Presses universitaires de Caen

Référence électronique

Christiane Marque-Pucheu, "Contribution des trois emplois de à la fin à la structuration du discours le temporel, l'aspectuel, le « reformulatif » », Discours [En ligne], 8 | 2011, mis en ligne le 11 juillet 2011, consulté le 01 mai 2019. URL : http://journals.openedition.org/discours/8468; DOI : 10.4000/ discours.8468

\section{(c) (i) (9)}

Discours est mis à disposition selon les termes de la licence Creative Commons Attribution - Pas d'Utilisation Commerciale - Pas de Modification 4.0 International. 

Revue de linguistique, psycholinguistique et informatique

Contribution des trois emplois de à la fin à la structuration du discours: le temporel, l'aspectuel, le « reformulatif »

Christiane Marque-Pucheu

EA 4089, Sens, Texte, Informatique, Histoire Université Paris-Sorbonne (Paris IV) 



\title{
Contribution des trois emplois de à la fin à la structuration du discours: le temporel, l'aspectuel, le « reformulatif»
}

\author{
Christiane Marque-Pucheu \\ EA 4089, Sens, Texte, Informatique, Histoire \\ Université Paris-Sorbonne (Paris IV)
}

\begin{abstract}
Nous distinguons trois fonctionnements de à la fin dans des suites de phrases coordonnées ou juxtaposées, Po P1, où P1 comporte l'adverbial à la fin. Dans la première partie, nous opposons sur la base de propriétés (portée, type de phrase, position dans la phrase), un emploi temporel (à la fiñ) un emploi aspectuel (à la fin2) et un emploi pragmatique (à la finz) exprimant une attitude du locuteur qui intervient pour mettre fin à un état de fait. Dans la deuxième partie, nous montrons comment, malgré ces différences, les trois emplois servent la structuration du discours. À la finı introduit une énumération externe (en impliquant un balancement avec au début); à la finz introduit une énumération interne, la répétition du procès dans $\mathrm{Po}$ ayant pour résultat un autre procès dans $\mathrm{P}_{1}$, ce que traduisent la paraphrase avec finir par et la possibilité d'introduire une relation de subordination consécutive entre Po et $P_{1}$; à la finz vise à agir sur l'allocutaire pour qu'il mette un terme à un dire ou un faire (finissons-en) exprimé ou non dans Po.
\end{abstract}

Mots clés: adverbial, aspect, cadre temporel, conséquence, énonciation, grammaticalisation, portée, pragmaticalisation

We can distinguish three functions of à la fin in the second parts of coordinated and juxtaposed phrases, $P_{0}, P_{1}$ in which $P_{1}$ contains the adverbial à la fin. We first compare three uses of the adverbial on the basis of qualities (scope, type of sentence, position in the sentence): a temporal use (à la fin1), an aspectual use (à la fin2) and a pragmatic use (à la fin3) expressing an attitude on the part of the speaker who intervenes to put an end to a state of affairs. We then show how, in spite of these differences, the three uses contribute to structuring the discourse. À la fin1 introduces an external enumeration (implying opposition to au début); à la finz introduces an internal enumeration, the repetition of the process in $P_{0}$ that results in another process in $P_{1}$ which can be translated by the paraphrase finir par and the possibility of introducing a relation of consecutive subordination between Po and P1; à la finz aims to act on the addressee so that he ends the speech or action (finissons-en) expressed or not in Po.

Keywords: adverbial, aspect, temporal framework, consequence, enunciation, grammaticalization, scope, pragmaticalization

Nous la sèmerons à la fin du mois de Février, ou commencement de Mars. (O. de Serres, Le Théâtre d'agriculture et mesnage des champs, I603, t. II)

À la fin tu es las de ce monde ancien.

(Apollinaire, Alcools, г913) 
Tant va la cruche à l'eau qu'à la fin elle se casse. (proverbe)

Levez-vous à la fin! Faites quelque chose.

(V. Leduc, La Bâtarde, i964)

\section{Introduction ${ }^{1}$}

Il est bien connu qu'un adverbial de cadre temporel peut avoir une autre utilisation. Nef (1980), par exemple, a montré comment maintenant connaissait un emploi temporel, c'est-à-dire fonctionnait comme marqueur de structuration globale (Maintenant, il bat sa femme et prostitue ses enfants), et un emploi non temporel (Maintenant, moi ce que je t'en dis c'est pour toi) où il notait un commentaire énonciatif (Ducrot, 1980 $)^{2}$. Cette distinction entre temporel et non temporel concerne aussi enfin qui peut introduire le dernier d'une série ou exprimer une réprobation, et à la fin comme dans [I] et [3] ou [3a] :

[I] À la fin (du repas) un des invités est parti.

$=$ À l'issue ( $\neq$ au début $)$ du repas un des invités est parti

[2] Un des participants a réussi à la fin.

= Un des participants a réussi finalement.

[2a] Déjà depuis longtemps je tâche à le comprendre, Et si plus je l'écoute, et moins je puis l'entendre: Je vois bien à la fin que je m'en dois mêler.

= Je vois bien finalement que je m'en dois mêler.

(Molière, Sganarelle ou le Cocu imaginaire, 1660)

[2b] Ces clowns fatiguent à la fin.

= Ces clowns fatiguent finalement.

$\left(\right.$ GlossaNet $\left.^{3}\right)$

[3] C’en est trop, à la fin!

= C'en est trop, enfin!

(Molière, L'Étourdi ou les Contre-temps, 1663)

[3a] Des sangliers, toujours des sangliers, merde à la fin, il faut changer un peu!!! = Des sangliers, toujours des sangliers, merde enfin, il faut changer un peu!!! (GlossaNet)

1. Au départ, cet article doit beaucoup, pour le meilleur, à Danielle Leeman. Je tiens aussi à remercier mes relecteurs pour la finesse de leurs remarques.

2. Voir aussi Jollin-Bertocchi, 2003.

3. Moteur de recherche spécialisé: http://glossa.fltr.ucl.ac.be. 
Mais si les études consacrées spécifiquement à enfin sont légion ${ }^{4}$, à la fin a fait couler moins d'encre, à l'exception de Poibeau et Maurel (1995), qui se placent dans l'optique d'une reconnaissance automatique. Certains dictionnaires ${ }^{5}$ se limitent généralement à la bipartition entre temporel dans [I] et énonciatif dans [3] et [3a]. Gross (1986) recense un autre emploi, observable dans [2], [2a] et [2b]. Nous désignerons désormais ces trois emplois successifs respectivement par à la finı, $\grave{a}$ la finz, à la finz.

À ces différences sémantiques se superposent des différences de propriétés concernant la portée, la position dans la phrase et le type de phrase (partie I). Notre hypothèse sera que, en dehors du premier emploi, qui est conjonctif/temporel et structure donc le discours, les deux autres peuvent également constituer des marqueurs de structuration. Pour le démontrer, nous examinerons notamment le contexte à gauche de la phrase comportant à la fin, ainsi que des paraphrases possibles, dans lesquelles un terme est en relation morphologique avec fin (partie 2).

\section{Différences de propriétés}

\subsection{Portée}

Dik (1997) présente les différents niveaux de portée qu'on peut observer dans la proposition ${ }^{6}$. Un premier niveau correspond à une spécification interne, l'adverbe indiquant comment le procès est mis en ouvre (manière, quantification, aspect): en l'occurrence, l'adverbe est supprimable mais n'est pas déplaçable, et difficilement focalisable par c'est... que.

[4] Luc m'amuse beaucoup.

Dans l'exemple [5], qui illustre le deuxième niveau, la prédication est étendue et consiste en une spécification externe où le procès est repéré au regard du temps $(\text { bier })^{7}$. Dik ne fait pas mention d'un niveau intermédiaire où le constituant est supprimable, focalisable et déplaçable, mais pas en tête de phrase, par exemple à la main dans Elle lave son linge fin à la main, analysable donc comme un modifieur (ou ajout) du SV, alors que hier ci-dessous en [5] serait modifieur ou ajout de phrase $^{8}$. Autrement dit, on aurait un niveau i qui correspond à un ajout de verbe,

4. Voir les références chez Buchi et Städtler, 2008.

5. Le nouveau Petit Robert, Dictionnaire encyclopédique Quillet.

6. Cela reprend un certain nombre de travaux sur le français: ceux de Mordrup (1976) sur les adverbes (ou expressions adverbiales), qui a bien distingué aussi différents types d'adverbes et justifié son classement par un ensemble de propriétés syntaxiques, ainsi que ceux de Molinier et Levrier (2000).

7. La spécification peut aussi relever de l'espace, de la cause ou de la conséquence.

8. Selon les conventions habituelles, SV désigne un syntagme verbal et SP, un syntagme prépositionnel. Par ailleurs, Po Pi désignera une suite de deux phrases coordonnées ou juxtaposées, où Pi comporte l'adverbial à la fin. 
un niveau 2 non mentionné par Dik (1997) qui correspond à un ajout de SV et un niveau 3 qui correspond à un ajout de phrase comme bier:

[5] Hier, il pleuvait.

Une différence de statut sépare le complément (de manière, quantifieur), relevant de [4] et le complément (circonstanciel de temps) relevant de [5] : dans [4], l'événement est qualifié, alors que dans [5], il est situé, recevant une détermination extérieure (Golay, 1959). Le niveau suivant, qui ne correspond plus aux constituants de la phrase, mais concerne l'extra-phrastique, est celui de l'évaluation propositionnelle par le locuteur qui qualifie le dit:

[6] Il pleuvra peut-être.

Enfin, le dernier niveau correspond à l'énoncé proféré où la manière de parler est qualifiée [7], ce qu'on peut paraphraser par sincèrement (parlant):

[7] Sincèrement, ce n'est pas une bonne affaire.

Ducrot (1980) avait déjà évoqué ce point en distinguant les «adverbes d'énonciation».

Reprenons la série [I] à [3a] donnée en introduction. Dans [I], un invité est parti à la fin (du repas), le SP à la fin est supprimable, focalisable, déplaçable en tête de phrase; il répond à la question quand? et localise (dans le temps) l'événement «un invité est parti».

En revanche, en [2], le SP à la fin, certes supprimable et déplaçable, ne répond pas (dans l'interprétation considérée) à la question quand?, bien qu'il marque la fin du procès comme précédemment; c'est qu'en fait, son rôle n'est pas uniquement un rôle de localisation temporelle. Nous pouvons mettre en évidence une portée sur le verbe, à la finz fonctionnant comme marque aspectuelle, en posant une équivalence à la fin = «finir par». «Finir par» marque la réussite ultime au terme d'une série d'efforts. L'aspect est donc de l'ordre du perfectif [+ télique] puisqu'au terme de la durée du procès on aboutit à un résultat:

[2'] Un des participants a fini par réussir.

On peut émettre l'hypothèse d'une portée double de à la finz $2^{9}$, qui porterait à la fois sur un constituant et sur la phrase. Comme finalement, à la fin refuse le test de l'extraction:

* C’est finalement qu'un des participants a réussi.

Cela indique l'absence de tout lien avec le verbe et un fonctionnement comme conjonctif. Mais on ne peut pas exclure:

9. Pour un autre exemple de portée double, voir Marque-Pucheu, 20II (à paraitre). 
C'est à la fin (d'une série d'efforts) qu'un des participants a réussi.

Caractérisons maintenant la portée du troisième emploi représenté par les exemples de la série [3]. Syntaxiquement, à la finz peut entrer dans une paraphrase où il modifie un verbe de dire sous-jacent (placé dans une phrase supérieure): je le (répète + dis) à la fin. Mais la paraphrase proposée ne rend pas compte de la subjectivité (profond agacement du locuteur) dont est chargée l'expression, qui serait plutôt de l'ordre d'une injonction cachée, où à la finz équivaudrait à finissons-en. Sémantiquement, l'expression implique le locuteur en traduisant un rapport (d'impatience ou d'agacement) avec son destinataire, ce que reflète l'intonation exclamative ${ }^{10}$.

Cependant, si l'expression à la finz peut constituer un énoncé autonome dans l'exemple suivant:

Je les voyais bien les autres, Maud Machin-Chouette et Denise Trucmuche et Nadine Je-ne-sais-pas-quoi, les amies de Maman que tu...

- Kim, à la fin!

(C. de Rivoyre, Les Sultans, 1964)

des dialogues courants plus naturels nécessitent un contexte:

Ça suffit à la fin!

Non mais c'est pas fini à la fin ?!

Arrêtez, merde, à la fin!

Autrement dit, il faut expliciter un minimum ce sur quoi porte à la fin. Et d'une manière générale, les occurrences de à la fin 3 s'insèrent dans des contextes assez larges:

[...] chez les Dambreville, sa main était chaude, il y avait des claques dans l'air. Alors, à toute volée, elle gifla Berthe.

Tiens! Tu m'embêtes, à la fin! ... quel pot!

(É. Zola, Pot-bouille, 1882)

Cette caractéristique justifie sa distinction avec l'emploi 2, en dépit d'une communauté de valeur (voir 2.2.2) et même si ce processus n'est sans doute pas engagé aussi fortement que pour d'autres expressions comme Au feu! ou Merde!

10. La frontière catégorielle entre un adverbial (traduisant un sentiment du locuteur) et une interjection divise lexicographes et linguistes. Le Dictionnaire encyclopédique Quillet évoque une «locution interjective marquant l'impatience», Le nouveau Petit Robert, une «locution adverbiale familière», le Trésor de la langue française, une «locution prépositive», "souvent en exclamation»; Gross (1986: 97-Ioo) en fait un adverbe, reconnaissant dans son ouvrage, à propos d'autres unités, qu'une distinction entre adverbe et interjection n'est pas toujours triviale. 


\subsection{Position dans la phrase et autres propriétés syntaxiques}

\subsection{1. À la fin1}

L'emploi I s'observe en position initiale ou finale, avec la réserve suivante: en position initiale, il constitue le cadre de l'énoncé (Charolles, 1997). En position finale, il instaure un recadrage - c'est-à-dire que à la fin appartient au focus/rhème de la phrase -, de telle sorte qu'on perçoit après coup que l'information énoncée concerne la fin d'un événement. Ainsi, dans l'exemple [I], bien que l'information un des invités est parti s'applique à la fin (du repas), on ne peut pas considérer qu'il ait équivalence totale avec l'exemple [I] comportant à la fin (du repas) en position finale, mais seulement une équivalence partielle notée $\cong$ dans [I'] :

[I] À la fin (du repas) un des invités est parti.

[’’] $\cong$ Un des invités est parti à la fin (du repas).

Malgré ce recadrage, les deux positions ne modifient donc pas la valeur.

Il faut toutefois distinguer dans ce premier emploi, d'une part, à la fin ( $d u$ repas), adverbial de cadre temporel qui peut alterner avec au début et au milieu et, d'autre part, à la fin, adverbial d'ordre et de rang (Molinier et Levrier, 2000: 57) comme dans:

Au début ils s'appréciaient, à la fin ils se sont brouillés.

Ce dernier n'a pas de complément explicité, il peut alterner avec au début, mais pas avec au milieu:

Au début ils s'appréciaient ( ${ }^{*}$ au milieu ils s'observaient), à la fin ils se sont brouillés.

Dans ce dernier cas, la position finale est possible pour à la fin, sous réserve d'un contraste établi avec un SP également en position finale:

?? Au début ils s'appréciaient, ils se sont brouillés à la fin.

Ils s'appréciaient au début, ils se sont brouillés à la fin.

À la fin (comme au début d'ailleurs), et plus précisément l'article défini, est anaphorique si le nom n'a pas de complément (de type $d u$ repas): on comprend qu'il s'agit de la fin de quelque chose (état ou événement), et donc le SP est compositionnel par rapport au sens «terme» de fin. La préposition à marque une localisation ponctuelle précise, elle pourrait ici commuter avec vers; vers la fin marquerait alors l'approximation. Le SP peut être soumis à restriction: C'est à la fin que... Ce n'est qu'à la fin que... et il est soumis à la portée de la négation ou de l'interrogation:

Ils ne se sont pas brouillés à la fin (mais dès le début).

Est-ce qu'ils se sont brouillés à la fin (ou dès le début)? 
Cela contribue à étayer la différenciation entre les trois emplois tout en justifiant le rassemblement opéré pour l'emploi i. Car malgré la différence de type anaphorique, nous avons choisi d'effectuer un regroupement sous à la fini. Dans le vers d'Apollinaire cité en exergue, il peut s'agir de l'interprétation temporelle (= à la fin de ce voyage, de ma vie, etc.); mais, placée en tête, l'expression joue avec les autres acceptions possibles de à la fin.

\subsection{2. À la fin2}

Rappelons les exemples de la série [2] ${ }^{11}$ :

[2a] Déjà depuis longtemps je tâche à le comprendre, Et si plus je l'écoute, et moins je puis l'entendre: Je vois bien à la fin que je m'en dois mêler.

(Molière, Sganarelle ou le Cocu imaginaire, 1660)

[2b] Ces clowns fatiguent à la fin.

(GlossaNet)

Ils montrent une position finale. La position initiale dans [2a'] et [2b'] va introduire une ambiguité, voire une préférence pour l'interprétation temporelle, c'est-à-dire à l'issue de $N$, et non plus aspectuelle, c'est-à-dire où l'on pouvait établir une synonymie avec finalement, à force, à la longue, ou encore tout compte fait, en définitive, en fin de compte:

[2a'] À la fin je vois bien que je m'en dois mêler.

[2b'] À la fin ces clowns fatiguent.

Nous justifions l'interprétation temporelle préférentielle observée dans les exemples précédents, par la structuration possible du discours que réalise le balancement à la fin... (mais pas) au début comme dans [2a"] et [2b”] :

[2a”] À la fin je vois bien que je m’en dois mêler, mais pas au début.

[2b”] À la fin ces clowns fatiguent, mais pas au début.

Le déplacement frontal de cet emploi 2, où à la fin est synonyme de finalement et où l'adverbial spécifie dans quelles conditions le procès est mis en œuvre ou

11. Le Dictionnaire du moyen français (DMF) (http://atilf.atilf.fr/dmf) date l'emploi 2, synonyme de finalement, de 1350 : À la fin. «Finalement»: "Et ot Melusigne, les deux ans après, deux filz, de quoy le premier ot a nom Fromont, et ama moult l'eglise, car bien le monstra a la fin, car il fu rendu moine a Malieres» (Jean d'Arras, Mélusine, env. 1392-I393). "[...] a la fin il fut trompé par l'orde eaue que l'amant de sa dicte femme getta par une fenestre sur elle» (Les Cent Nouvelles Nouvelles, env. I456-I467). "Et soyés aussy seur que de la mort que, se vous voulés longuement guerroier le roy, il sera à la fin trouvé par tout le monde que vous avés abusé du mestier de la guerre» (Jean Le Clerc, Interpolations et variantes de la Chronique scandaleuse, env. I5O2). 
évalué (= à la suite d'un constat), peut donc faire basculer dans l'emploi I comme dans [2a'] et [2b'], où l'événement est situé et où l'adverbial porte sur l'ensemble verbe/actants et non pas sur le procès. On opposera ainsi:

Il a réussi (à la fin $=$ à la longue).

$\neq$ À la fin il a réussi.

D’une manière générale (Le Querler, 1993: I83-I84), la position initiale favorise l'interprétation d'une portée phrastique comme dans l'emploi I, alors que les autres positions, notamment la position finale, favorisent l'interprétation d'une portée intra-prédicative (interne à un constituant) comme dans l'emploi 2.

L'interprétation peut donc changer selon la position ou, du moins, être ambiguë.

Toutefois, même si la position est finale, une structuration au début/au commencement... à la fin, fera privilégier l'interprétation temporelle, comme dans:

Chez nous, intervient le pauvre Méridional, les repas de fêtes sont si longs, que le pain, frais au commencement, est rassis à la fin!

(H. Barbusse, Le Feu, 1916)

\subsection{3. À la fin3}

Sauf effet de focalisation, c'est-à-dire si à la finz est interprété comme emphatique, le déplacement frontal est difficile dans l'emploi 3 :

[3’] ?? À la fin, c'en est trop!

Pourtant, tous les exemples du Dictionnaire encyclopédique Quillet des années cinquante, présentent à la fin en position initiale. À l'inverse, tant la base Frantext que les moteurs de recherche Google ou GlossaNet privilégient la position finale. Mentionnons les exemples suivants trouvés sur Google ${ }^{12}$ :

[3b] C'est vrai à la fin!

[3c] Y’en a marre à la fin!

dans lesquels la position initiale est moins acceptable:

[3b'] ?? À la fin, c'est vrai!

[3c'] À la fin, y'en a marre!

ou encore $[3 \mathrm{~d}]$ et $[3 \mathrm{e}]$ dans Frantext:

12. À l'adresse suivante: http://forum.wordreference.com/showthread.php?t=2043217 (site consulté le $\mathrm{I}^{\mathrm{er}}$ février 20II). 
[3d] Peureusement, l'autre s'était réfugié derrière la fontaine, en criant:

- C'est assommant, à la fin, si je ne peux plus vous adresser une observation, sans que celui-là se mette entre nous! ... j’ai bien voulu l'accepter [...].

(É. Zola, Pot-bouille, 1882)

Vous vous fichez de moi. Me prenez-vous pour un escroc? Et puis j'en ai plein le dos, à la fin.

(G. Courteline, Le Commissaire est bon enfant, 1900)

[3e] Toujours faire appel à leurs parties basses, à leur bêtise et à leur ignorance. - jamais un reproche! C'est assommant, à la fin! Dit le domestique.

(J. Renard, Journal: 1887-1910, 1910)

La position initiale semble donc perdre du terrain ${ }^{13}$. Ce cas de figure contredit donc l'orientation admise d'ordinaire, selon laquelle le passage à une valeur énonciative s'accompagne d'un affranchissement syntaxique (Combettes, 2003: I54).

Le comportement des trois à la fin au regard de la position dans la phrase ne met pas en évidence de différence sensible, si ce n'est une tendance à la position finale observée pour à la finz.

\subsection{Types de phrases}

\subsection{1. À la fin1}

Les conjonctifs, et notamment les adverbes de cadre temporel, admettent le type déclaratif, ce qui est de fait le cas de à la finı:

Au début ils s'appréciaient, à la fin ils se sont brouillés.

Les adverbes qui instaurent un cadre pour une proposition donnée admettent toujours le type interrogatif pour la proposition correspondante :

À la fin, il est parti. / Il est parti à la fin.

À la fin est-il parti? / Est-il parti à la fin?

De même, les impératives sont compatibles avec les adverbes de cadre (Molinier et Levrier, 2000: 36):

13. Cela le distingue nettement de l'adverbe enfin, même si, comme ce dernier, à la fin traduit le mécontentement du locuteur devant des propos ou une attitude et peut commuter avec lui dans cet exemple de Rossari (1994: 49):

Mais qu'est-ce que tu veux enfin?

Mais qu'est-ce que tu veux à la fin?

et même si, comme lui, il peut former une phrase (voir 2.1.3):

Enfin!

À la fin! 
À la fin, pars. / Pars à la fin. / Ne pars qu’à la fin.

La position frontale devant une phrase négative est également un critère de caractérisation:

À la fin, ils ne se sont pas brouillés. / À la fin, il n'est pas parti. / À la fin, surtout ne pars pas!

\subsection{2. À la fin2}

Avec à la finz, on n'observe de restriction ni sur les phrases déclaratives, ni sur les phrases négatives:

À la fin (à ta grande surprise) tu n'es pas las de ce monde ancien.

ni sur les phrases interrogatives:

Est-ce qu'il voit bien à la fin qu'il doit s'en mêler?

ni sur les impératives:

Comprends bien à la fin que tu dois t'en mêler ${ }^{14}$.

\subsection{3. À la fin3}

Sauf intonation particulière (exclamative), le type déclaratif est incompatible avec à la finz. L'exemple

?? Il ferme cette porte à la fin.

Il ferme cette porte à la fin!

ne peut correspondre qu'à l'emploi 2 avec un contexte gauche comme

Le froid commence à envahir la pièce.

De même que certains adverbes de commentaire énonciatif, à la finz peut figurer dans des phrases de type interrogatif:

La fermeras-tu cette porte à la fin?

Est-ce qu'il va la fermer cette porte à la fin?

L'impératif est également compatible avec le commentaire énonciatif:

Ferme-la cette porte, à la fin!

Tais-toi à la fin!

14. Mais les caractéristiques du verbe peuvent interdire le type impératif comme dans:

??* Réussis à la fin!

En effet, sauf scénario particulier, le sujet de réussir ne peut pas être agentif:

* Il a réussi exprès.

Toutefois, cette restriction étant générale avec l'impératif, elle ne saurait être prise en compte dans la distinction. 
Ainsi, pour à la finz, l'intonation exclamative marquant l'impatience se superpose systématiquement aux autres types (interrogatif, impératif), alors que ce n'est pas le cas pour à la finı et à la finz.

Les types de phrases permettent donc de distinguer surtout le troisième emploi des deux autres.

\subsection{Interprétation sémantique}

L'emploi I ressortit aux adverbiaux temporels, d'ordre et de rang, du moins quand le complément de nom est omis (voir I.2.I). Quand le complément est présent ou que l'omission du nom variable s'accompagne d'un «effet elliptique pronominal» (Gross, 1986: 188), il s'agit d'un adverbial de temps qui peut noter des dates plus ou moins variées (à la fin du mois/de l'année 20IO) ou indiquer un segment de durée (à la fin de la réunion).

La question de la distinction sémantique se pose surtout pour les emplois 2 et 3, d'autant que le contexte gauche peut comporter dans les deux cas un marqueur consécutif (si... que, tellement... que) et qu'ils peuvent tous deux être paraphrasés par finir par (voir 2.I.2 et 2.I.3). Un exemple comme

Il m'ennuie à la fin!

est ambigu, sauf ponctuation ou intonation exclamative nette. Il peut être interprété comme un emploi 2 (en [8]) ou un emploi $3^{15}$ (en [9]):

[8] Il m'ennuie finalement / en définitive / à la longue / Il finit par m’ennuyer

[9] Il m'ennuie (enfin + merde alors)!

Toutefois, l'emploi 3 (en [9]) ne peut s'interpréter que «négativement», marquant un agacement. Sur ce point, il se distingue d'ailleurs de enfin, qui peut s'interpréter tant négativement (reproche, agacement) que positivement (soulagement après attente):

Tu la fermes cette porte (enfin + à la fin)!

Quant à l'emploi 2, il peut indifféremment être associé à un verbe «négatif» ou «positif»:

Ce compositeur m'ennuyait à la fin.

Ce compositeur me séduisait à la fin.

Un autre critère de différenciation entre les deux est le fait que à la finz n'est pas compatible avec un état:

15. Des caractéristiques particulières devraient être mentionnées: prosodiques (évoquées indirectement avec l'exclamation) et gestuelles. 
(Enfin $+{ }^{*}$ à la fin), tu es là, il me tardait de te voir.

Alors ça vient, oui, tu arrives à la fin? / Ah chouette, enfin te voilà! Tu arrives à la fin!

alors que cette interdiction n'existe pas avec à la finz:

Il était las à la fin. = Il finissait par être las à la fin.

Malgré ces différences, nous montrons qu'il s'agit de marqueurs de structuration dans les trois cas.

\section{Trois marqueurs de structuration différents}

Soit Pr la phrase comportant à la fin et Po la phrase précédente, VI et Vo étant respectivement les verbes figurant dans $\mathrm{P}_{\mathrm{I}}$ et $\mathrm{Po}$ :

[Io] (Po) Il est arrivé au début de la séance. $(\mathrm{PI})$ Il est parti à la fin.

[II] (Po) Il s'y est repris à plusieurs fois. $(\mathrm{PI}) \mathrm{Il}$ a réussi à la fin.

[I2] (Po) Arrête de te plaindre. (PI) C'en est trop à la fin!

\subsection{Contexte gauche $(\mathrm{Po})$}

Les liens avec le contexte gauche se manifestent différemment.

\subsection{1. À la fin1}

Selon qu'un complément peut le suivre ou non, à la fin I est adverbe de cadre temporel strict (alternant avec au milieu et au début) ou conjonctif marquant l'ordre et le rang (voir I.2.I) et s'opposant à au début ${ }^{16}$ :

[13] La réunion a commencé à 20 heures. Le vote a eu lieu au milieu. Luc est arrivé à la fin (de la réunion).

[i4] Au début ils s'appréciaient, à la fin ils se sont brouillés.

Quel que soit le cas, le contexte gauche (Po) peut faire mention d'un événement (la réunion a commencé), d'un état, d'un sentiment (ils s'appréciaient) ou d'une action. Dans Pı, un autre événement/état ou action intervient (Luc est arrivé, Ils se sont brouillés) quand le premier événement/état s'achève. Une paraphrase possible de Pi dans [13] est:

[I3’] Luc est arrivé quand la réunion (finissait + était finie).

16. La valeur référentielle est marquée ou non par ce complément de nom: La réunion a commencé à 20 heures. Il est arrivé à la fin ( $\mathrm{E}+$ de la réunion). 
Elle peut mettre en évidence un rapport de succession (était finie) entre Po et PI ou d'inclusion (finissait). Dans [I4], la paraphrase [I4'] indique un rapport de succession entre Po et $\mathrm{PI}$, à comprendre «dans le temps de leur relation».

[I4'] Ils se sont brouillés quand ils ont eu fini de s'apprécier.

\subsection{2. À la fin2}

Contrairement au cas précédent, le contexte gauche impose une contrainte aspectuelle: Po doit être recouvert par le procès. Soit le procès est duratif, c'est-à-dire a le trait [-momentané] ${ }^{17}$ :

On t'a attendu des heures, et à la fin heureusement, tu es arrivé.

Soit le procès ne l'est pas, c'est-à-dire a le trait [+momentané] et dans ce cas, il doit être répété pour couvrir la durée:

Luc a longtemps hésité: il est entré et ressorti (plusieurs fois $+{ }^{*}$ une fois $\left.+{ }^{*} \mathrm{E}\right)$. Et puis à la fin il est resté.

D'une manière générale, si l'on cherche à compléter les exemples [2] et [2a] en ajoutant un contexte gauche, on s'aperçoit que ce dernier doit comporter des indications de répétition comme plusieurs fois (en [2]) ou de durée comme depuis que dans l'exemple de Molière (en [2a]) ${ }^{18}$ :

[2] (Po) Un des participants a essayé plusieurs fois. ( $\mathrm{PI})$ Il a réussi à la fin / ?? (Po) Un des participants a essayé. $(\mathrm{PI})$ Il a réussi à la fin.

[2a] (Po) Déjà depuis longtemps je tâche à le comprendre,

Et si plus je l'écoute, et moins je puis l'entendre:

$(\mathrm{PI})$ Je vois bien à la fin que je m'en dois mêler.

(Molière, Sganarelle ou le Cocu imaginaire, 1660)

La même analyse pourrait s'appliquer à cet exemple de Flaubert où plusieurs actions se succèdent ${ }^{19}$ :

17. Le test correspondant est la possibilité d'accepter un verbe de phase (commencer).

18. Dans Marque-Pucheu (1995), nous avons mis en évidence un phénomène apparenté, mais la contrainte de répétition imposée par au fil de $N$ se limitait à la phrase correspondante et n'affectait pas obligatoirement le contexte gauche.

19. Dans cet exemple de Philippe de Vigneulles (Les Cent Nouvelles Nouvelles, 1515):

[...] ilz misrent leur reliquiaire au jeu et juerent pleuseurs partyes dessus, et tellement que à la fin tout leur tourna mal, et leur fuit force de se rendre, car ilz n'avoient plus gaige ne argen.

La séquence «à la fin tout leur tourna mal» fait suite à «juerent pleuseurs partyes», donc à une action répétée. De même, chez Jean de La Taille en 1573 dans Les Corrivaus, «le voicy à la fin» fait écho à «celuy que je cherche», où chercher est un procès ayant le trait [-momentané] (voir 2.1.2):

Mais voicy venir à la bonne heure celuy que je cherche.

Claude et Filadelfe 
[...] Morel lui-même s'en mêla, y usa son éloquence, y compromit sa dialectique et y réussit à la fin, ce qui l'étonna beaucoup lui-même, car il n'eut pas autant de mal qu'il l'avait cru d'abord, et Henry se laissa facilement convaincre.

(G. Flaubert, La Première Éducation sentimentale, 1845)

Le Dictionnaire encyclopédique Quillet propose, comme synonymes de à la fin, entre autres, à la longue et à force. Les deux expressions commutent bien dans Pr. Mais à force peut aussi être introduit dans Po, corroborant le fait que la durée doit être recouverte:

À force d'essayer il a réussi.

Cette paraphrase illustre le lien de nature consécutive entre Po et $\mathrm{PI}$, les occurrences répétées conduisant à $\mathrm{P}_{\mathrm{I}}$. Mais elle n'introduit pas de terme morphologiquement apparenté à fin. Or, nous souhaiterions corréler aux propriétés syntaxiques et sémantiques, des arguments de type morphologique (voir l'introduction). Une paraphrase possible pour je vois bien à la fin que je dois m'en mêler [2a], tenant compte de cette contrainte, serait je finis par voir que je dois m'en mêler. Il existe malgré tout un équivalent commutable qui comporte le mot fin: en fin de compte. Notons encore je décide de mettre fin, je fais en sorte que ça prenne fin.

Alors que le contexte gauche (Po) de à la finı introduit une succession, celui de à la finz instaure une relation consécutive, comme le confirme l'exemple ci-après, où nous avons introduit de telle sorte que:

C'est vrai que j'ai plaqué l'turbin. Mais l'ouvrier' gagn' pas son pain; quoi qu'a fasse, alle est mal payée, a n'fait mêm' pas pour son loyer: [de telle sorte que] à la fin, quoi, ça décourage, on n'a pus de cœur à l'ouvrage, ni le caractère ouvrier.

(J. Rictus, Le Cour populaire, 1914)

À la finz se distingue des deux autres fonctionnements.

\subsection{3. À la fin3}

Examinons le contexte gauche (Po) de à la finz observable dans PI. Soit les dialogues:

[I5] (Po) - Range ta chambre.

- Oui, je vais le faire.

- Tout de suite, j’ai dit.

- Attends un peu encore.

$(\mathrm{PI})$ - Range ta chambre à la fin.

Claude. Le voicy à la fin, Dieu mercy.

Filadelfe. Et bien Claude, quelles nouvelles?

Claude. - Fort bonnes. Voicy le jour que vous avez le plus desiré.

Plus rarement, l'information se trouve dans le contexte droit. Ainsi, chez René de Lucinge (Dialogue du François et du Savoysien, 1593):

[...] le peuple se lassera à la fin soubs le fardeau de nos guerres [...].

Mais l'analyse reste identique. La répétition est portée par le pluriel du substantif prédicatif «guerres». 
[ı] (Po) - On a sonné

- Qui c'est?

- C'est le facteur

- Tu crois?

(PI) - Va ouvrir à la fin.

Le contexte gauche (Po) fait apparaittre une non-action/attitude (refus de ranger [I5]; ne pas ouvrir [16]). À la finz traduit alors une requête en vertu de laquelle ladite (non-)action doit prendre fin ${ }^{20}$ et en vertu de laquelle, le cas échéant, une autre doit commencer. Parfois, le contexte gauche (Po) n'est pas explicite et $\mathrm{P}_{\mathrm{I}}$ se limite à un nom apostrophé $(\text { Kim })^{21}$ :

(P0) Je les voyais bien les autres, Maud Machin-Chouette et Denise Trucmuche et Nadine Je-ne-sais-pas-quoi, les amies de Maman que tu...

(P1) - Kim, à la fin!

(C. de Rivoyre, Les Sultans, 1964)

L'adverbial sert alors à couper la parole : c'est le simple fait de parler qui doit prendre fin, l'utilisation de à la fin invitant Kim à se taire. $\grave{A}$ la fin 3 instaure donc une rupture dans le discours en cherchant à mettre fin à une (non-)action ou à une (non-)parole.

À leur manière, les trois emplois introduisent une étape, mais en s'opposant les uns aux autres: $\grave{a}$ la fin I marque une succession, à la finz une conséquence et à la finz une rupture. Les comparaisons deux à deux qui suivent, vont confirmer cette opposition.

\subsection{Vérification par une comparaison des emplois deux à deux}

\subsection{1. À la fin1 et à la fin2: des énumératifs différents}

Conjonctif temporel, à la finı est sémantiquement un adverbial «d'ordre et de rang» (Molinier et Levrier, 200o); faisant écho à au début, il structure explicitement le discours :

Au début il échouait tout le temps. Mais il a fini par réussir à la fin ${ }^{22}$.

Cette possibilité est moins claire avec à la finz. Est-ce à dire que dans ce deuxième emploi, à la fin n'est pas un marqueur de structuration? Au lieu de faire écho à au

20. L'apparentement avec enfin apparâtt clairement. Rossari (1994: 27-28) cite: «Enfin! [inséré dans un contexte où un professeur entre dans une classe indisciplinée]», l'adverbe étant considéré comme un «connecteur reformulatif» supposant implicitement: Cessez ce chabut.

21. Parfois le contexte immédiat (il est mort) ne permet pas d'établir une déduction. Dans la réplique : Il est mort, à la fin!, où à la fin traduit l'agacement, la mise en évidence se fait par la portée sur un verbe de dire: Je le (dis + répète) à la fin.

22. L'association ni contradictoire ni redondante entre finir par et à la fin indique que c'est donc à la finI qui est ici réalisé. 
début, il marque un résultat après une série de faits non nécessairement énumérés; il est compatible avec longtemps, souvent, ce que n'est pas à la finI:

Il a longtemps essayé, et il a bien fait car il a réussi à la fin (= finalement).

Il ne peut s'agir de à la fin I puisque longtemps et souvent n'impliquent pas une «fin», ce qui précisément rend à la finı inacceptable, comme l'indique le contexte gauche. En effet, il a réussi à la fin suppose après plusieurs essais:

Il a essayé (plusieurs fois), il a réussi à la fin.

Contrairement à à la finı, à la fin z n'est donc pas un énumératif strict, mais un énumératif plus implicite en fonction du type de procès apparaissant dans $\mathrm{Po}$, car il suppose une réitération des occurrences du verbe (il a essayé plusieurs fois) ou leur vision «massive» (longtemps). Cette énumération implicite conduit à un résultat exprimé par le verbe de PI. La paraphrase mettant en jeu finir par indique cette caractéristique résultative:

$\mathrm{Il}$ a réussi à la fin. $=\mathrm{Il}$ a fini par réussir.

Inversement, cette possibilité d'équivalence n'existe pas avec à la finI:

Il est parti à la fin (du repas). $\neq$ Il a fini par partir (à la fin du repas).

De même, des marqueurs consécutifs comme tellement que ne sont pas pertinents dans l'emploi I (exemple [I7]), alors qu'ils caractérisent l'emploi 2 (exemple [I8] ${ }^{23}$ :

[17] Au début ils s’appréciaient. À la fin ils se sont brouillés. $\leftrightarrow$ ?? Au début ils s'appréciaient tellement quà la fin ils se sont brouillés.

[I8] Il a essayé plusieurs fois. Il a réussi à la fin. $\leftrightarrow \mathrm{Il}$ a tellement essayé qu'il a réussi (à la fin).

23. Historiquement, les marqueurs consécutifs sont très fréquemment cooccurrents de à la fin, qu'ils prennent la forme de tellement que [19], si que [20], tant que [21], à force de [22] :

[19] [...] il ne trouva point sa hacquenée, et encore plus quand il n'en oyoit point de nouvelles un jour, deux jours, un mois, deux mois, trois mois; tellement qu'à la fin il fut contraint d'achepter une mule. (Bonaventure Des Périers, Nouvelles récréations et joyeux devoirs, 1558)

[20] Mais, pour toutes ses excuses, n'appaisa poinct le roy d'Angleterre, lequel le prochassa si très instamment, que, à la fin, le procureur l'eust à sa requeste, et retourna en sa maison [...].

(Marguerite de Navarre, L'Heptaméron, 1550)

[21] Tant va le pot à l'eau qu'il brise

Tel est loué qui peu se prise:

Tant vente, qu'il pleut à la fin.

(Jean-Antoine de Baï, Mimes, enseignements et proverbes: second livre, 1581)

[22] À la fin, à force d'impétuosité, pressée du remords de la conscience, leur demanda congé d'aller à Romme [...].

(Marguerite de Navarre, L'Heptaméron, 1550) 
Cependant, la possibilité de paraphrase en finir par n'est pas spécifique de à la finz, elle existe également avec à la finz:

C'en est trop, à la fin! = Ça finit par être trop!

Il s'agit donc maintenant de distinguer à la finz et à la finz, qui partagent cette caractéristique aspectuelle résultative.

\subsection{2. À la finz et à la fin3: résultatif vs résultatif performatif}

À la finz peut instaurer un début. Par exemple, dans il m'énerve à la fin, on peut introduire un verbe de phase, ce qui donne il commence à m'énerver à la fin. Mais cette caractéristique ne vaut pas pour tous les exemples mettant en jeu à la finz:

Il a hésité longtemps, il s'est décidé à la fin ( $\neq$ il a commencé à se décider).

De plus, cela ne permet pas de le distinguer de l'emploi 3, puisqu'une paraphrase de à la finz mettant également en jeu un verbe de phase initiale est possible ${ }^{24}$ :

Tu m'énerves, à la fin. $=$ Tu commences à m'énerver.

Il s'agit bien d'un emploi 3 puisqu'on intime l'ordre d'arrêter. La différence est alors essentiellement performative. D'une part, les verbes associés à à la finz sont rarement agentifs, ce que corrobore la difficulté à observer un impératif, alors que les verbes associés à à la finz 3 peuvent être agentifs, et par suite, être utilisés à l'impératif. De plus, la relation consécutive se traduit par le fait qu'elle fait intervenir le locuteur: aide-moi à la fin résulte d'un agacement, alors que dans le cas de à la finz, la relation consécutive était simplement constatée par le locuteur qui ne visait pas à agir sur l'allocutaire. Enfin, à la fin 3 traduit toujours un sentiment, ce qui n'est pas nécessairement le cas avec à la finz, qui peut n'être qu'un simple constat, comme dans il a réussi à la fin. Et par rapport aux autres emplois, à la finz ne nécessite pas de contexte linguistique dans $P_{I}$ et peut même constituer une réplique seule, comme dans l'exemple de C. de Rivoyre que nous rappelons:

(P0) Je les voyais bien les autres, Maud Machin-Chouette et Denise Trucmuche et Nadine Je-ne-sais-pas-quoi, les amies de Maman que tu...

(P1) - Kim, à la fin!

(C. de Rivoyre, Les Sultans, 1964)

Paradoxalement, à la finz initierait le discours: Ouvrez à la fin!, qui vise à faire agir l'interlocuteur peut entraîner une réponse: Oui, j'arrive. Cet emploi règle donc la conversation et, par suite, le discours, forçant l'allocutaire à agir à l'inverse de ce qu'il faisait.

24. Voir, en anglais, you're beginning to get on my nerves ou you're beginning to annoy me. 


\section{Conclusion}

Malgré la tripartition effectuée sur la base de propriétés propres à chacun d'eux, nous montrons qu'il est possible d'établir une filiation (temporelle) entre les trois emplois et, qu'à leur manière, ils structurent le discours. Dans une suite Po Pı, où Pi comporte à la fin, à la finı marque une étape, impliquant un balancement avec au début, à la finz un résultat, ce qu'indiquent la paraphrase en finir par et la possibilité d'introduire une relation consécutive entre Po et Pr. Le dernier emploi, à la finz, structure les interactions langagières en mettant un terme à une (non-)attitude/action. Plus précisément, le contexte gauche des énoncés comportant à la finz décrit un état, un sentiment ou une action qui se répète ou dure et dont la répétition ou la durée finit par entraîner un autre état, sentiment ou action. Le contexte linguistique ou situationnel des énoncés comportant à la finz décrit ou suppose implicitement un état, un sentiment ou une action qui se répète ou dure et dont la répétition ou la durée finit par entraîner un sentiment d'agacement chez le locuteur, déclenchant à la finz: en proférant à la finz, le locuteur vise alors à mettre fin à cette répétition.

Un point de départ strictement temporel (à la finI) permet donc de passer à une relation «logique», consécutive (à la finz), puis à une relation pragmatique (à $\left.\operatorname{la}_{\text {fin }}\right)^{25}$, donc d'une valeur référentielle (temps) à une valeur grammaticale et aspectuelle, puis à une valeur pragmatique. Ce parcours, conforme à ce que prédit la «Théorie de la grammaticalisation», devrait être confirmé par une étude diachronique.

\section{Références}

Buchi, É. et STÄDTLER, T. 2008. La pragmaticalisation de l'adverbe enfin du point de vue des romanistes ( $E n f i n$, de celui des francisants qui conçoivent leur recherche dans le cadre de la linguistique romane»). In J. Durand, B. Habert et B. Laks (eds), Actes $d u$ Congrès mondial de linguistique française ( $C M L F$ 2008). Paris: Institut de linguistique française: I59-I7I.

Charolles, M. 1997. L'encadrement du discours - univers, champs, domaines et espaces. Cabiers de recherche linguistique $6: \mathrm{I}-73$.

Combettes, B. 2003. Les marqueurs de topicalisation en français: un cas de grammaticalisation. In B. Combettes, C. Schnedecker et A. Theissen (eds), Ordre et distinction dans la langue et le discours. Actes du colloque international de Metz. Paris: Champion: I49-167.

Dik, S.C. 1997. Theory of Functional Grammar, K. Hengeveld (ed.). Berlin - New York: Mouton de Gruyter. Partie I: The Structure of the Clause.

Dostie, G. 2004. Pragmaticalisation et marqueurs discursifs. Analyse sémantique et traitement lexicographique. Champs linguistiques. Bruxelles: De Boeck-Duculot.

Ducrot, O. 1980. Les mots du discours. Paris: Éditions de Minuit.

Golay, J.-P. 1959. Le complément de manière est-il un complément circonstanciel? Le Français moderne I: 65-7I.

25. Voir également l'exemple de parallèlement (Marque-Pucheu, 2003). 
Gross, M. 1986. Grammaire transformationnelle du français. 3- Syntaxe de l'adverbe. Malakoff: Cantilène.

Hansen, M.-B.M. 2008. Particles at the Semantics/Pragmatics Interface: Synchronic and Diachronic Issues. A Study with Special Reference to the French Phasal Adverbs. Current Research in the Semantics/Pragmatics Interface i9. Amsterdam - New York - Paris : Elsevier.

JoLLIN-BERTOCCHI, S. 2003. La polyvalence de l'adverbe maintenant. L'Information grammaticale $97: 26-30$.

Le Querler, N. I993. Les circonstants et la position initiale. In C. GuImIER (ed.), IOOI circonstants. Caen: Presses universitaires de Caen : 159-184.

Marque-Pucheu, C. 1995. Variété des marques syntaxiques et lexicales de l'aspect: au fil de N. Linguisticae Investigationes XIX (2): 369-385.

Marque-Pucheu, C. 2003. Après un point on mène un parallèle: parallèlement ou l'articulation logique et chronologique du discours. In B. Combettes, C. SchnedeckeR et A. Theissen (eds), Ordre et distinction dans la langue et le discours. Actes du colloque international de Metz. Paris: Champion: 317-333.

Marque-Pucheu, C. 201 I (à paraître). Contraintes et figement(s): des compléments nucléaires et périphériques aux énoncés situationnels. Dossier présenté pour l'obtention de l'habilitation à diriger des recherches, Paris-Sorbonne.

Molinier, C. et Levrier, F. 2000. Grammaire des adverbes. Description des formes en -ment. Genève - Paris: Droz.

MoRDRup, O. 1976. Une analyse non transformationnelle des adverbes en -ment. Revue Romane, numéro spécial II. Copenhague: Akademisk Forlag.

NEF, F. I980. Maintenant I et maintenant2: sémantique et pragmatique de «maintenant» temporel et non temporel. In J. DAvid et R. MARTin (eds), La notion d'aspect. Actes du colloque de Metz, I8-20 mai 1978. Recherches linguistiques 5. Paris : Klincksieck: I45-165.

Poibeau, T. et Maurel, D. 1995. À la fin de, préposition ou déterminant complexe dans les adverbiaux de temps? Cabiers de grammaire 20: IOI-III.

Rossari, C. 1994. Les opérations de reformulation. Analyse du processus et des marques dans une perspective contrastive français-italien. Berne - New York: Peter Lang.

\section{Dictionnaires et corpus}

ATILF, base textuelle Frantext.

ATILF, Dictionnaire du moyen français $\left(\mathrm{DMF}_{2}\right)$. Accessible en ligne sur http://stella.atilf. fr/gsouvay/dmf2 (cf. http://www.atilf.fr/dmf pour DMFI).

Dubois, J. et Dubois-Charlier, F. 2004. Locutions en français. Aix-en-Provence: chez les auteurs.

Mortier, R. (dir.) i953. Dictionnaire encyclopédique Quillet. Paris: A. Quillet.

Moteur de recherche Google.

Moteur de recherche GlossaNet: http://glossa.fltr.ucl.ac.be.

Rey, A. et Rey-Debove, J. (dir.) 1994. Le nouveau Petit Robert. Paris: Dictionnaires Le Robert. 